\title{
DEFORMATION THEORY OF SUBSPACES IN A RIEMANN SPACE
}

\author{
V. HLAVATÝ
}

Summary. This paper deals with the infinitesimal transformation $(2,1)$ of a family $\left(V_{m}\right)$ of subspaces $V_{m}$ in a Riemann space. In $\$ \S 1-4$ the transformation $(2,1)$ is applied on internal objects of a $V_{m}$, while in $\S \S 5$ and 6 the characteristic mixed tensors $K_{a_{r} \cdots a_{1}}^{\nu}$ (cf. the equation $(1,2 ; 4))$ of a $V_{m}$ are investigated with respect to $(2,1)$. Finally, some applications of the theory are given in $\$ \$ 7$ and 8 . In particular the statement expressed by the equation $(8,10) \mathrm{b}$ is the generalization of the well known Levi-Civita result for $m=1$, while the statement expressed by the equation $(8,10) c$ generalizes the classical result (for $m=1, n=2$ ) by Jacobi.

\section{Preliminary.}

(1) Let $V_{n}$ be a $n$-dimensional Riemann space $(n \geqq 2)$, referred to the real coördinate system $\xi^{p},{ }^{1} g_{\lambda \mu}=g_{\mu \lambda}$ its metric tensor, $\Gamma_{\lambda \mu}^{\nu}=\Gamma_{\mu \lambda}^{\nu}$ the corresponding Christoffel symbols and $\nabla_{\mu}$ the covariant derivative operator in $V_{n}$ with respect to $\Gamma_{\lambda \mu}^{\nu}$. The curvature tensor of $\Gamma_{\lambda \mu}^{\nu}$ will be denoted by

$$
R_{\omega \mu \lambda}^{\prime} \equiv \partial_{\mu} \Gamma_{\lambda \omega}^{\prime}-\partial_{\omega} \Gamma_{\lambda \mu}^{\nu}+\Gamma_{\alpha \mu}^{\prime} \Gamma_{\lambda \omega}^{\alpha}-\Gamma_{\alpha \omega}^{\prime} \Gamma_{\lambda \mu}^{\alpha} \quad\left(\partial_{\mu} \equiv \frac{\partial}{\partial \xi^{\mu}}\right) .
$$

(2) Let $V_{m}$ be a $m$-dimensional subspace of $V_{n}(1 \leqq m<n)$ referred to the real parameters ${ }^{1} \eta^{a}$ and let

$$
\xi^{p}=\xi^{\nu}\left(\eta^{1}, \cdots, \eta^{m}\right)
$$

be its parametric equations. ${ }^{2}$ Throughout this paper we consider only the case where the matrix of the mixed tensor

$$
T_{a}^{\prime} \equiv \partial_{a} \xi^{\prime}
$$

$$
\left(\partial_{a} \equiv \frac{\partial}{\partial \eta^{a}}\right)
$$

is of rank $m$. Hence the metric tensor ${ }^{3}$

$$
g_{a b}=g_{b a}=g_{\lambda \mu} T_{a b}^{\lambda \mu}
$$

Received by the editors May 31, 1949 and, in revised form, July 11, 1949.

${ }^{1}$ Greek (Latin) indices run from $\dot{i}$ to $\dot{n}$ (from 1 to $m$ ).

${ }^{2}$ Any function considered in this paper is understood to be a real and continuous one, as well as its derivatives which appear in the discussion.

${ }^{3}$ Here and later on we put $T_{a_{1} \ldots a v}^{\lambda \ldots \nu} \equiv T_{a_{1} \ldots}^{\lambda} T_{a_{v}}^{\nu}, v=2,3, \cdots$. 
is of rank $m$ and consequently the corresponding Christoffel symbols $\Gamma_{a b}^{c}=\Gamma_{b a}^{c}$ exist. The curvature tensor of $V_{m}$ will be denoted by

$$
P_{d c b}^{a} \equiv \partial_{c} \Gamma_{b d}^{a}-\partial_{d} \Gamma_{b c}^{a}+\Gamma_{h c}^{a} \Gamma_{b d}^{h}-\Gamma_{h d}^{a} \Gamma_{b c}^{h} .
$$

Considering the connections $\Gamma_{\lambda \mu}^{\nu}$ and $\Gamma_{b c}^{a}$ we may introduce three different kinds of covariant derivatives, namely

$(1,2 ; 3 a) \quad \nabla_{\mu} V_{\lambda}^{\prime \cdots} \ldots \partial_{\mu} V_{\lambda}^{\alpha \cdots}+\Gamma_{\alpha \mu}^{\nu} V_{\lambda}^{\alpha \cdots}+\cdots-\Gamma_{\lambda \mu}^{\alpha} V_{\alpha}^{\prime \cdots} \cdots-\cdots$,

for a tensor field defined over $V_{n}$,

$(1,2 ; 3 \mathrm{~b}) \nabla_{a} V_{\lambda}^{\nu \cdots} \equiv \partial_{a} V_{\lambda}^{\nu \cdots}+T_{a}^{\mu}\left\{\Gamma_{\alpha \mu}^{\nu} V_{\lambda}^{\alpha \cdots}+\cdots-\Gamma_{\lambda \mu}^{\alpha} V_{\alpha \cdots}^{\nu \cdots}-\cdots\right\}$,

for a tensor field defined over $V_{m}$ and

$$
D_{b} V_{a \cdots}^{\nu \cdots} \equiv \partial_{b} V_{a \cdots}^{\nu \cdots}+\Gamma_{\lambda \mu}^{\nu} T_{b}^{\mu} V_{a \cdots}^{\lambda \cdots}+\cdots-\Gamma_{a b}^{c} V_{c}^{\nu \cdots}-\cdots
$$

for a mixed tensor field defined over $V_{m}$. The embedding theory of a $V_{m}$ in a $V_{n}$ may be described by means of the mixed tensors $T_{a}^{\nu}$ and

$$
K_{a_{r}}^{\nu} \cdots a_{1} \equiv D_{a_{r}} \ldots D_{a_{2}} T_{a_{1}}^{\prime}, \quad r=2, \cdots, N,
$$

where $N$ is the number of osculating spaces of $V_{m}{ }^{4}$

Incidentally $K_{a b}^{\nu}=K_{b a}^{\nu}$ lies with its index $\nu$ in the first normal space of $V_{m}$.

(3) A family $\left(V_{m}\right)$ of a set of $V_{m}$ 's is defined as a set of $V_{m}$ 's such thiat through any generic point of the $V_{n}$ there is only one element $V_{m}$ of $\left(V_{m}\right)$. Each element $V_{m}$ of the family $\left(V_{m}\right)$ is referred to the parameter system $\eta^{a}$ and we keep the parameter transformation $\eta^{a^{\prime} \leftrightarrow} \eta^{a}$ independent of the coördinate system $\xi^{\nu}$. Hence

$$
\partial_{\mu} \frac{\partial \eta^{a^{\prime}}}{\partial \eta^{a}}=0 \quad \partial_{\mu} \frac{\partial \eta^{a}}{\partial \eta^{a^{\prime}}}=0 .
$$

On the other hand, an object $\Omega$ defined over $\left(V_{m}\right)$ is defined also over $V_{n}$ and consequently may be thought of as expressed either by means of the $\eta^{a}$ or by means of the $\xi^{\nu}$. This is in particular true for $T_{a}^{\nu}, g_{a b}, \Gamma_{a b}^{c}$. Whenever we apply on such an object the operator $\partial_{\mu}$ it is understood that we consider it expressed by means of the $\xi^{\nu}$. Taking in account $(1,3 ; 1)$ we see that ${ }^{5} \partial_{\mu} g_{a b}, \partial_{\mu} \Gamma_{a b}^{c}, \nabla_{\omega} T_{a}^{\nu}$ are

${ }^{4}$ Cf. V. Hlavatý: Embedding theory of $a W_{m}$ in a $W_{n}$ (to be published in Actualités Scientifiques et Industrielles) where the case of a Riemann space is included. Cf. also J. A. Schouten and E. R. van Kampen, Über die Krümmung einer $V_{m}$ in $V_{n}$; eine Revision der Krümmungstheorie (Math. Ann. vol. 105, p. 144-159).

${ }^{5} \nabla_{\omega} T_{a}^{\nu} \equiv \partial_{\omega} T_{a}^{\nu}+\Gamma_{\lambda \omega}^{\nu} T_{a}^{\lambda}$ according to $(1,2 ; 3 \mathrm{a})$, while (cf. $\left.(1,2 ; 3 \mathrm{~b})\right) \nabla_{b} T_{a}^{\nu} \equiv \partial_{b} T_{a}^{\nu}$ $+\Gamma_{\lambda \omega}^{\nu} T_{a b}^{\lambda \omega}$. 
tensors (resp. vectors) not only with respect to the coördinate transformation but also with respect to the parameter transformation. Let $P$ be a generic point of $V_{n}$ and let $V_{m}$ be the element of $\left(V_{m}\right)$ containing $P$. If a tensor field $T$ defined over $V_{n}$ has the property that $T(P)$ is in the tangential space (or in some of the normal spaces) of the $V_{m}$ already mentioned, then we say that the field $T$ is tangent (normal) to $\left(V_{m}\right)$.

2. Fundamental definitions. Let $V^{v}=V^{v}(\xi)$ be the components of a contravariant vector field given over $V_{n}$ and let

$$
{ }^{*} \xi=\xi+\epsilon V^{\nu} \quad(\epsilon \rightarrow 0 \text { is a constant })
$$

be the infinitesimal transformation of a Lie group with the generator $V^{\lambda} \partial_{\lambda}$. If a generic point $P(\xi)$ describes a subspace $V_{m}$ of the family $\left(V_{m}\right)$ then the point ${ }^{*} P \equiv P\left({ }^{*} \xi\right)$ describes a subspace ${ }^{*} V_{m}$. We shall assume throughout this paper that the set of all ${ }^{*} V_{m}$ constructed in this way is a family, which we denote by $\left({ }^{*} V_{m}\right)$. In the following definition $X=X(P)$ denotes a tensor field (a set of tensor fields) defined either over $\left(V_{m}\right)$ or over $V_{n}$ and $F[X] \equiv F(\xi)$ is a function of $X$.

Definition $(2,1) . X^{\prime}$ is the value of $X$ in ${ }^{*} P, X^{\prime} \equiv X\left({ }^{*} P\right),{ }^{\circ} X$ is the tensor at ${ }^{*} P$ which one gets by parallel displacement in $V_{n}$ of $X(P)$ from $P$ to ${ }^{*} P$. If $X$ is defined over $\left(V_{m}\right)$, then ${ }^{*} X$ is the tensor field (a set of tensor fields) defined over $\left({ }^{*} V_{m}\right)$ in the same way as $X$ is defined over $\left(V_{m}\right)$. If $X$ is defined over $V_{n}$, then ${ }^{*} X \equiv X^{\prime}$. Furthermore

$$
{ }^{*} F \equiv F\left[{ }^{*} X\right], \quad{ }^{\circ} F \equiv F\left[{ }^{\circ} X\right], \quad F^{\prime} \equiv F\left({ }^{*} \xi\right)
$$

in the first case and

$$
{ }^{*} F \equiv F^{\prime} \equiv F\left({ }^{*} \xi\right), \quad{ }^{\circ} F \equiv F\left[{ }^{\circ} X\right]
$$

in the second case.

We use these symbols in the following definition of the symbols $\tau$, $\omega$, and $\Delta$ :

Definition $(2,2)$. The operators $\tau, \omega, \Delta$ are defined by the following equations
(a) $\tau F \equiv \lim _{\epsilon \rightarrow 0} \frac{{ }^{*} F-{ }^{\circ} F}{\epsilon}$
(translation of $F)$,
(b) $\omega F \equiv \lim _{\epsilon \rightarrow 0} \frac{F^{\prime}-{ }^{\circ} F}{\epsilon}$
(variation of $F$ ),
(c) $\Delta F \equiv(\tau-\omega) F$
(deformation of $F$ ).

In this paper we shall investigate the application of these operators 
on the objects of $\left(V_{m}\right)$.

3. The fields $T_{a}^{y}$ and $g_{a b}$.

Theorem $(3,1)$. We have
(a) $\tau T_{a}^{\prime}=\nabla_{a} V^{\prime}$,
(b) $\omega T_{a}^{\prime}=V^{\mu} \nabla_{\mu} T_{a}^{\prime}$,

(c) $\Delta T_{a}^{\prime}=L_{a}^{\prime}$,

where

$$
L_{a}^{\prime} \equiv \nabla_{a}^{\prime} V-V^{\mu} \nabla_{\mu} T_{a}^{\prime}=\partial_{a} V^{\prime}-V^{\prime \prime} \partial_{\mu} T_{a}^{\prime} .
$$

Proof. Let $X \equiv F[X] \equiv T_{a}^{\nu}$. Then

$$
\begin{gathered}
{ }^{*} F \equiv \frac{\partial^{*} \xi^{\nu}}{\partial \eta^{a}}=T_{a}^{\prime}+\epsilon \partial_{a} V^{\prime}, \quad F^{\prime} \equiv T_{a}^{\prime}\left({ }^{*} \xi\right)=T_{a}^{\prime}+\epsilon V^{\mu} \partial_{\mu} T_{a}^{\prime}+\cdots,{ }^{\circ} \\
{ }^{\circ} F \equiv{ }^{\circ} T_{a}^{\prime}=T_{a}^{\prime}-\epsilon V^{\mu} \Gamma_{\lambda \mu}^{\prime} T_{a}^{\lambda}+\cdots
\end{gathered}
$$

and consequently
(a) ${ }^{*} F-{ }^{\circ} F=T_{a}^{\nu}+\epsilon\left(\partial_{a} V^{\nu}+\Gamma_{\lambda \mu}^{\prime} T_{a}^{*} V^{\lambda}\right)-T_{a}^{\nu}+\cdots$,
(b) $F^{\prime}-{ }^{\circ} F=T_{a}^{\prime}+\epsilon V^{\mu}\left(\partial_{\mu} T_{a}^{\prime}+\Gamma_{\lambda_{\alpha}}^{\prime} T_{a}^{\lambda}\right)-T_{a}^{\prime}+\cdots$.

From $(3,3)$ ab we have $(3,1) a b$ and these equations together with $(3,2)$ lead to $(3,1)$ c.

Theorem $(3,2)$. We have

$$
\begin{aligned}
& \begin{array}{ll}
\text { (a) } \tau g_{a b}=2 W_{a b}{ }^{7} & \text { (b) } \omega g_{a b}=G_{a b} \text {, }
\end{array} \\
& \text { (c) } \Delta g_{a b}=2 g_{\lambda \mu} L_{(a}^{\lambda} T_{b)}^{\mu}
\end{aligned}
$$

where

$$
W_{a b} \equiv T_{(a b)}^{\lambda_{\mu}} \nabla_{\lambda} V_{\mu,} \quad G_{a b} \equiv V^{\mu \prime} \partial_{\mu} g_{a b} .
$$

Proof. Put $X \equiv g_{\lambda \mu}$. Then
(a) ${ }^{*} X \equiv X^{\prime}={ }^{*} g_{\lambda_{\mu}}=g_{\lambda_{\mu}}+\epsilon V^{\omega} \partial_{\omega} g_{\lambda_{\mu}}+\cdots$,
(b) ${ }^{\circ} X={ }^{\circ} g_{\lambda_{\mu}}=g_{\lambda \mu}+\epsilon V^{\omega}\left(\Gamma_{\lambda \omega}^{\prime} g_{\nu \mu}+\Gamma_{\mu \omega}^{\prime} g_{\lambda_{\nu}}\right)+\cdots={ }^{*} g_{\lambda \mu}$.

On the other hand, if we denote by $X$ the set of tensor fields $g_{\lambda \mu}$, $T_{a}^{\nu}$ we have for $F[X] \equiv g_{\lambda \mu} T_{a}^{\lambda} T_{b}^{\mu}=g_{a b}$ by virtue of $(3,6)$

- Throughout this paper the dots denote the coefficient of $e^{2}$.

7 Substantially equivalent formulas to $(3,1)$ a and $(3,4)$ a may be found also in the papers in the bibliography, which deal with the theory of deformation of subspaces from a different point of view. 


$$
\begin{aligned}
& \text { (a) }{ }^{*} F \equiv{ }^{*} g_{a b}={ }^{*} g_{\lambda \mu}{ }^{*} T_{a}^{\lambda} * T_{b}^{\mu}=\left(g_{\lambda \mu}+\epsilon V^{\omega} \partial_{\omega} g_{\lambda \mu}+\cdots\right) \\
& \cdot\left(T_{a}^{\lambda}+\epsilon \partial_{a} V^{\lambda}+\cdots\right)\left(T_{b}^{\mu}+\epsilon \partial_{b} V^{\mu}+\cdots\right) \\
& =g_{a b}+\epsilon\left[V^{\omega} T_{a}^{\lambda} T_{b}^{\mu} \partial_{\omega} g_{\lambda \mu}+2 g_{\lambda \mu} T_{(b}^{\mu} \partial_{a)} V^{\lambda}\right]+\cdots \\
& =g_{a b}+2 \epsilon T_{(a}^{\lambda} T_{b)}^{\mu} \nabla_{\lambda} V_{\mu}+\cdots \text {, } \\
& \text { (b) } F^{\prime} \equiv g_{a b}^{\prime}=g_{\lambda \mu}^{\prime} T_{a}^{\lambda^{\prime}} T_{b}^{\mu^{\prime}}=\left(g_{\lambda \mu}+\epsilon V^{\omega} \partial_{\omega} g_{\lambda \mu}+\cdots\right) \\
& \cdot\left(T_{a}^{\lambda}+\epsilon V^{\rho} \partial_{\rho} T_{a}^{\lambda}+\cdots\right)\left(T_{b}^{\mu}+\epsilon V^{\sigma} \partial_{\sigma} T_{b}^{\mu}+\cdots\right) \\
& =g_{a b}+\epsilon\left[V^{\omega} T_{a}^{\lambda} T_{b}^{\mu} \partial_{\omega} g_{\lambda \mu}+2 g_{\lambda \mu} V^{\omega}\left(\partial_{\omega} T_{(a)}^{\lambda}\right) T_{b)}^{\mu}\right]+\cdots \\
& =g_{a b}+\epsilon V^{\omega} \partial_{\omega} g_{a b}+\cdots \text {, } \\
& \text { (c) }{ }^{\circ} F \equiv{ }^{\circ} g_{a b}={ }^{\circ} g_{\lambda \mu}{ }^{\circ} T_{a}^{\lambda^{\circ}} T_{b}^{\mu}=\left[g_{\lambda \mu}+\epsilon V^{\omega}\left(\Gamma_{\lambda \omega}^{\nu} g_{\nu \mu}+\Gamma_{\mu \omega \omega \lambda \nu}^{\nu}\right)+\cdots\right] \\
& \cdot\left(T_{a}^{\lambda}-\epsilon V^{\rho} \Gamma_{\alpha \rho}^{\lambda} T_{a}^{\alpha}+\cdots\right)\left(T_{b}^{\mu}-\epsilon V^{\sigma} \Gamma_{\beta \sigma}^{\mu} T_{b}^{\beta}+\cdots\right) \\
& =g_{a b}+\epsilon\left[T_{a}^{\lambda} T_{b}^{\mu} V^{\omega}\left(\Gamma_{\lambda \omega}^{\nu} g_{\nu \mu}+\Gamma_{\mu \omega}^{\nu} g_{\lambda \nu}\right)-2 g_{\lambda \mu} T_{(b}^{\mu} T_{a)}^{\alpha} V^{\omega} \Gamma_{\alpha \omega}^{\lambda}\right] \\
& +\cdots=g_{a b}+\cdots \text {. }
\end{aligned}
$$

Hence

$$
\begin{aligned}
& \text { (d) }{ }^{*} F-{ }^{\circ} F \equiv{ }^{*} g_{a b}-{ }^{\circ} g_{a b}=2 \epsilon T_{(b}^{\mu} T_{a)}^{\lambda} \nabla_{\lambda} V_{\mu}+\cdots, \\
& \text { (e) } F^{\prime}-{ }^{\circ} F=g_{a b}^{\prime}-{ }^{\circ} g_{a b}=\epsilon V^{\omega} \partial_{\omega} g_{a b}+\cdots .
\end{aligned}
$$

These equations lead to $(3,4) \mathrm{ab}$ and from these we have $(3,4) \mathrm{c}$.

Definition $(3,1)$. The transformation $(2,1)$ is called a rigid translation if $\tau g_{a b}=0$.

Theorem $(3,3 \mathrm{a})$. Let $V^{v}$ be a tangential vector field to $\left(V_{m}\right)$. A necessary and sufficient condition that $(2,1)$ be a rigid translation is the (Killing) equation

$$
D_{(a} V_{b)}=0 .
$$

Proof. In our case we have ${ }^{8} V_{a}=V_{\lambda} T_{a}^{\lambda}, V_{\lambda}=V_{c} T_{\lambda}^{c}$ and consequently (by virtue of $\Gamma_{a b}^{c}=T_{\lambda}^{c} \nabla_{b} T_{a}^{\lambda}$ )

$$
\begin{aligned}
D_{b} V_{a} & =\partial_{b} V_{\lambda} T_{a}^{\lambda}-T_{\lambda}^{c}\left(\nabla_{b} T_{a}^{\lambda}\right) V_{c}=\left(\partial_{b} V_{\lambda}\right) T_{a}^{\lambda}+V_{\lambda} \partial_{b} T_{a}^{\lambda}-\left(\nabla_{b} T_{a}^{\lambda}\right) V_{\lambda} \\
& =T_{a}^{\nu}\left(\partial_{b} V_{\nu}-\Gamma_{\mu \nu}^{\lambda} T_{b} V_{\lambda}^{\mu}\right)=T_{b} T_{a}^{\mu} \nabla_{\mu}^{\lambda} V_{\lambda} .
\end{aligned}
$$

The proof follows from $(3,9),(3,5)$, and $(3,4) \mathrm{a}$.

Theorem $(3,3 \mathrm{~b})$. Let $V^{\prime}$ be a normal vector field to $\left(V_{m}\right)$. A necessary and sufficient condition that $(2,1)$ be a rigid translation is: $V^{\nu}$ is in the $x$ th normal space of $V_{m}, x=2,3, \cdots$.

${ }^{8} T_{\lambda}^{c} \equiv T_{a}^{v} g^{a c} g_{\nu \lambda}$. 
Proof. In our case we have $V_{\lambda} T_{a}^{\lambda}=0$ and consequently $(3,10) \quad T_{a}^{\lambda} T_{b}^{\mu} \nabla_{\lambda} V_{\mu}=-T_{a}^{\lambda} V_{\mu} \nabla_{\lambda} T_{b}^{\mu}=-\left(D_{a} T_{b}^{\mu}\right) V_{\mu}=-K_{a b}^{\mu} V_{\mu}$.

The tensor $K_{a b}^{\mu}$ lies with $\mu$ in the first normal space of $\left(V_{m}\right)$. By virtue of this fact, we get the theorem $(3,3 \mathrm{~b})$ from $(3,4) \mathrm{a}$ and $(3,5)$.

REMARK. The theorem $(3,3 \mathrm{~b})$ is the generalization of the well known fact that the "deformation" of the arc of a curve in a threedimensional Euclidean space along its binormal is equal to zero.

\section{The field $\Gamma_{a b}^{c}$.}

THEOREM $(4,1)$. We have
(a) $\tau \Gamma_{a b}^{c}=W_{a b}^{c} \equiv W_{a b d} g^{d c}$,
(b) $\omega \Gamma_{a b}^{c}=G_{a b}^{c}$,

$$
\text { (c) } \Delta \Gamma_{a b}^{c}=Q_{a b}^{c}
$$

where

$$
\begin{aligned}
\text { (a) } W_{a b d} & \equiv D_{a} W_{d b}+D_{b} W_{d a}-D_{d} W_{b a}, \\
\text { (b) } G_{a b}^{c} & =V^{\omega} \partial_{\omega} \Gamma_{a b}, \\
\text { (c) } Q_{a b}^{c} & \equiv W_{a b d} g^{d c}-G_{a b b}^{c} .
\end{aligned}
$$

Proof. First of all we have

$$
\partial_{a} W_{d b}+\partial_{b} W_{d a}-\partial_{d} W_{a b}=W_{a b d}+2 \Gamma_{b a}^{\bullet} W_{d e .}
$$

Furthermore, if $X$ is the set of tensors $g_{a b}, g^{c d}$, we get for $F[X] \equiv \Gamma_{a b}^{c}$ by virtue of $(3,7) \mathrm{d}$ and $(4,3)$

$$
\begin{aligned}
* F \equiv{ }^{*} \Gamma_{a b}^{c}= & 1 / 2^{*} g^{c d}\left(\partial_{a}{ }^{*} g_{b d}+\partial_{b}{ }^{*} g_{a d}-\partial_{d}{ }^{*} g_{a b}\right) \\
= & 1 / 2\left[{ }^{\circ} g^{c d}-2 \epsilon W^{c d}+\cdots\right]\left[\partial_{a}\left({ }^{\circ} g_{b d}+2 \epsilon W_{b d}+\cdots\right)\right. \\
& +\partial_{b}\left({ }^{\circ} g_{a d}+2 \epsilon W_{a d}+\cdots\right) \\
& \left.-\partial_{d}\left({ }^{\circ} g_{a b}+2 \epsilon W_{a b}+\cdots\right)\right] \\
= & { }^{\circ} \Gamma_{b a}^{c}+\epsilon\left\{{ }^{c} g^{c d}\left[W_{a b d}+2 \Gamma_{b a}^{e} W_{d e}\right]\right. \\
& \left.-2 W^{c d}{ }^{\circ} \Gamma_{b a}^{e}{ }^{o} g_{e d}\right\}+\cdots .
\end{aligned}
$$

On the other hand, we see from $(3,7) \mathrm{c}$ that

$$
{ }^{\circ} \Gamma_{b a}^{c}=\Gamma_{b a}^{c}+\cdots .
$$

From $(4,5),(4,4)$ we have $(4,1) a$. The equation $(4,1) \mathrm{b}$ follows from

$$
F^{\prime} \equiv \Gamma_{a b}^{c \prime}=\Gamma_{a b}^{c}+\epsilon V^{\omega} \partial_{\omega} \Gamma_{a b}^{c}+\cdots
$$

and $(4,5)$. The equation $(4,1) \mathrm{c}$ is obvious. 
Later on we shall need also the coefficients

$$
\Gamma_{a b}^{c}=1 / 2 g^{c d \prime}\left(\partial_{a} g_{b d}^{\prime}+\partial_{b} g_{a d}^{\prime}-\partial_{d} g_{a b}^{\prime}\right)
$$

which are related to $\Gamma_{a b}^{c \prime}$ by a relation introduced in the following lemma:

Lemma $(4,1)$. We have

$$
\Gamma_{a b}^{c}-\Gamma_{a b}^{c \prime}=\frac{\epsilon}{2} g^{c d}\left[L_{a}^{\omega} \partial_{\omega} g_{d b}+L_{b}^{\omega} \partial_{\omega} g_{d a}-L_{d}^{\omega} \partial_{\omega} g_{a b}\right]+\cdots
$$

Proof. We get from $(4,7)$ and $(3,7) b$

$$
\begin{aligned}
{ }^{c} \Gamma_{a b}^{c}= & 1 / 2\left(g^{c d}+\epsilon V^{\omega} \partial_{\omega} g^{c d}+\cdots\right)\left[\partial_{a}\left(g_{b d}+\epsilon V^{\rho} \partial_{\rho} g_{b d}+\cdots\right)\right. \\
& +\partial_{b}\left(g_{a d}+\epsilon V^{\rho} \partial_{\rho} g_{a d}+\cdots\right) \\
& \left.-\partial_{d}\left(g_{a b}+\epsilon V^{\rho} \partial_{\rho} g_{a b}+\cdots\right)\right] \\
= & \Gamma_{a b}^{c}+\frac{\epsilon}{2}\left\{2 \Gamma_{a b}^{h} g_{h d} V^{\rho} \partial_{\rho} g^{c d}+g^{c d}\left[\left(\partial_{a} V^{\rho}\right) \partial_{\rho} g_{b d}\right.\right. \\
& +\left(\partial_{b} V^{\rho}\right) \partial_{\rho} g_{a d}-\left(\partial_{c} V^{\rho}\right) \partial_{\rho} g_{a b} \\
& \left.\left.+g^{c d} V^{\rho}\left(\partial_{a} \partial_{\rho} g_{b d}+\partial_{b} \partial_{\rho} g_{a d}-\partial_{d} \partial_{\rho} g_{a b}\right)\right]\right\}+\cdots
\end{aligned}
$$

On the other hand

$$
\begin{aligned}
& \Gamma_{a b}^{c \prime}=\Gamma_{a b}^{c}+\epsilon V^{\rho} \partial_{\rho} \Gamma_{a b}^{c}+\cdots=\Gamma_{a b}^{c}+\epsilon\left[V^{\rho}\left(\partial_{\rho} g^{c d}\right) \Gamma_{a b}^{h} g_{h d}\right. \\
& \left.+1 / 2 g^{c d} V^{\rho}\left(\partial_{\rho} \partial_{a} g_{b d}+\partial_{\rho} \partial_{b} g_{a d}-\partial_{\rho} \partial_{d} g_{a b}\right)\right]+\cdots, \\
& \left(\partial_{a} \partial_{\rho}-\partial_{\rho} \partial_{a}\right) g_{b d}=\left(T_{a}^{\lambda} \partial_{\lambda} \partial_{\rho}-\left(\partial_{\rho} T_{a}^{\lambda}\right) \partial_{\lambda}-T_{a}^{\lambda} \partial_{\rho} \partial_{\lambda}\right) g_{b d} \\
& =-\left(\partial_{\rho} T_{a}^{\lambda}\right)\left(\partial_{\lambda} g_{b d}\right) \text {. }
\end{aligned}
$$

Comparing $(4,9),(4,10)$, and $(4,11)$, we obtain

$$
\begin{aligned}
{ }^{\prime} \Gamma_{a b}^{c}-\Gamma_{a b}^{c}= & \frac{\epsilon}{2} g^{c d}\left[\left(\partial_{a} V^{\rho}-V^{\lambda} \partial_{\lambda} T_{a}^{\rho}\right) \partial_{\rho} g_{b d}+\left(\partial_{b} V^{\rho}-V^{\lambda} \partial_{\lambda} T_{b}^{\rho}\right) \partial_{\rho} g_{a d}\right. \\
& \left.-\left(\partial_{d} V^{\rho}-V^{\lambda} \partial_{\lambda} T_{d}^{\rho}\right) \partial_{\rho} g_{a b}+\cdots\right]
\end{aligned}
$$

and this equation gives us at once $(4,8)$.

5. The field $K_{b a}^{\nu} \cdot{ }^{9}$ The first theorem concerns the translation of the

- Throughout $\$ \$ 5$ and 6 we shall sometimes make use of a special coördinate (parameter) system and shall symbolize the statement: " in the special coördinate (parameter) system $\Gamma_{\lambda \mu}^{\nu}=0\left(\Gamma_{b a}^{c}=0\right)$ at $P^{n}$ by using the symbol $\doteq, \Gamma_{\lambda \mu}^{\nu} \doteq 0\left(\Gamma_{a b}^{c} \doteq 0\right)$. An equation with $\doteq$ has to be understood as valid in a special coördinate (parameter) system at $P$ where both last equations hold. On the other hand, the sign $=$ does not impose any restriction whatsoever on the choice of the coördina te (parameter) system. 
tensor

$$
K_{b a}^{\prime}=K_{a b}^{\prime} \equiv D_{b} T_{a}^{\prime} .
$$

TheOREM $(5,1)$. The following equation holds:

$$
\tau K_{b a}^{\prime}=D_{b} D_{a} V^{\nu}-V^{\omega} T_{b a}^{\lambda \mu} R_{\omega \mu \lambda}^{\nu}-W_{b a}^{c} T_{c}^{\nu} .
$$

Proof. First of all we have according to $(2,2) \mathrm{b}$ for $X \equiv g_{\lambda_{\mu}}, g^{\lambda \nu}$, $F[X] \equiv F(\xi)=\Gamma_{\lambda \mu}^{\nu}$

$$
{ }^{*} \Gamma_{\lambda \mu}^{\prime}=\Gamma_{\lambda \mu}^{\prime \prime} \doteq \epsilon V^{\omega} \partial_{\omega} \Gamma_{\lambda \mu}^{\prime}+\cdots
$$

and moreover by virtue of $(4,4)$ and $(4,5)$

$$
* \Gamma_{b a}^{c} \doteq \epsilon W_{b a}^{c}+\cdots \text {. }
$$

Hence

$$
\begin{aligned}
{ }^{*} K_{b a}^{\nu}= & { }^{*} D_{b}^{*} T_{a}^{\nu} \doteq \partial_{b}\left(T_{a}^{\nu}+\epsilon \partial_{a} V^{\nu}+\cdots\right) \\
& +\epsilon V^{\nu}\left(\partial_{\rho} \Gamma_{\lambda \mu}^{\nu}\right)\left(T_{b}^{\mu}+\epsilon \partial_{b} V^{\mu}+\cdots\right)\left(T_{a}^{\lambda}+\epsilon \partial_{a} V^{\lambda}+\cdots\right) \\
& -\epsilon W_{b a}^{c}\left(T_{c}^{\nu}+\epsilon \partial_{c} V^{\nu}+\cdots\right)
\end{aligned}
$$

and consequently

$$
\begin{aligned}
{ }^{*} K_{b a}^{\prime} \doteq & \partial_{b} T_{a}^{\nu}+\epsilon\left\{\partial_{b} \partial_{a} V^{\nu}+V^{\rho}\left(\partial_{\rho} \Gamma_{\lambda \mu}^{\prime}\right) T_{b a}^{\mu \lambda}-W_{b a}^{c} T_{c}^{\nu}\right\}+\cdots \\
\doteq & D_{b} T_{a}^{\nu}+\epsilon\left\{D_{b} D_{a} V^{\nu}-V^{\omega}\left[\partial_{\mu} \Gamma_{\omega \lambda}^{\nu}-\partial_{\omega} \Gamma_{\mu \lambda}^{\nu}\right] T_{b a}^{\mu \lambda}-W_{b a}^{c} T_{c}^{\prime}\right\} \\
& +\cdots \\
\doteq & D_{b} T_{a}^{\prime}+\epsilon\left\{D_{b} D_{a} V^{\nu}-V^{\omega} T_{b a}^{\mu \lambda} R_{\omega \mu \lambda}^{\nu}-W_{b a}^{c} T_{c}^{\nu}\right\}+\cdots
\end{aligned}
$$

On the other hand

$$
{ }^{\circ} K_{b a}^{\prime} \doteq D_{b} T_{a}^{\prime}+\cdots=K_{b a}^{\nu}+\cdots .
$$

The equations $(5,4) \mathrm{b}$ and $(5,4) \mathrm{c}$ lead to $(5,2)$ with the $\doteq$ sign. This equation, being a tensor equation valid for a special coördinate (and parameter) system at a generic point $P$, holds for all coördinate (and parameter) systems at a generic point.

The second theorem concerns the variation of $K_{a b}^{\prime}$ :

THEOREM (5,2). The following equation holds:

$$
\omega K_{b a}^{\prime}=V^{\omega}\left[D_{b} \nabla_{\omega} T_{a}^{\nu}-T_{b a}^{\mu \lambda} R_{\omega \mu \lambda}^{\nu}+\left(\nabla_{\mu} T_{a}^{\nu}\right)\left(\nabla_{\omega} T_{b}^{\mu}\right)\right]-G_{b a}^{c} T_{c}^{\nu} .
$$

Proof. First of all we have 


$$
\begin{aligned}
\partial_{\omega} \partial_{b} T_{a}^{\nu}-\partial_{b} \partial_{\omega} T_{a}^{\nu} & =\partial_{\omega} T_{b}^{\mu} \partial_{\mu} T_{a}^{\nu}-T_{b}^{\mu} \partial_{\mu} \partial_{\omega} T_{a}^{\nu}=\left(\partial_{\omega} T_{b}^{\mu}\right)\left(\partial_{\mu} T_{a}^{\nu}\right) \\
& \doteq\left(\nabla_{\omega} T_{b}^{\mu}\right)\left(\nabla_{\mu} T_{a}^{\nu}\right) \\
D_{b} \nabla_{\omega} T_{a}^{\nu} & \doteq \partial_{b} \partial_{\omega} T_{a}^{\nu}+T_{b a}^{\mu \lambda}\left(\partial_{\mu} \Gamma_{\lambda_{\omega}}^{\nu}\right)
\end{aligned}
$$

Consequently

$$
\begin{aligned}
K_{b a}^{\prime \prime} \equiv & \left(D_{b} T_{a}^{\prime}\right)^{\prime}=K_{b a}^{\prime}+\epsilon V^{\omega} \partial_{\omega} K_{b a}^{\nu}+\cdots \\
\equiv & K_{b a}^{\prime}+\epsilon V^{\omega}\left[\partial_{\omega} \partial_{b} T_{a}^{\prime}+\left(\partial_{\omega} \Gamma_{\lambda \mu}^{\prime}\right) T_{b a}^{\mu \lambda}-\left(\partial_{\omega} \Gamma_{a b}^{c}\right) T_{c}^{\prime}\right]+\cdots \\
= & K_{b a}^{\nu}+\epsilon V^{\omega}\left\{\left[D_{b} \nabla_{\omega} T_{a}^{v}+T_{b a}^{\mu \lambda}\left(\partial_{\omega} \Gamma_{\lambda \mu}^{\prime}-\partial_{\mu} \Gamma_{\lambda \omega}^{\prime}\right)\right.\right. \\
& \left.\left.+\left(\nabla_{\omega} T_{b}^{\mu}\right)\left(\nabla_{\mu} T_{a}^{\nu}\right)\right]-G_{b a}^{c} T_{c}^{\nu}\right\} .
\end{aligned}
$$

The proof follows from $(5,6)$ and $(5,4) c$.

Theorem $(5,3) . P u t$

$$
L_{b a}^{\nu} \equiv D_{b} L_{a}^{\nu}+L_{b}^{\mu} \nabla_{\mu} T_{a}^{\nu}-Q_{b a}^{c} T_{c}^{\nu} .
$$

Then

$$
\begin{aligned}
L_{b a}^{\nu}= & D_{b} L_{a}^{\nu}-V^{\omega} T_{b a}^{\mu \lambda} R_{\omega \mu \lambda}^{\nu}-Q_{b a}^{c} T_{c} \\
& +\left(T_{b}^{\mu} \nabla_{\mu} V^{\omega} \nabla_{\omega}-V^{\omega} \nabla_{\omega} T_{b}^{\mu} \nabla_{\mu}\right) T_{a}^{\nu}
\end{aligned}
$$

and

$$
\Delta K_{b a}^{\nu}=L_{b a}^{\nu}
$$

Proof. We have from $(5,2)$ and $(5,5)$

$$
\begin{aligned}
\Delta K_{b a}^{\prime} & =(\tau-\omega) K_{b a}^{\nu}=D_{b} D_{a} V^{\nu}-V^{\omega} D_{b} \nabla_{\omega} T_{a}^{\nu} \\
& -V^{\omega}\left(\nabla_{\omega} T_{b}^{\mu}\right) \nabla_{\mu} T_{a}^{k}-Q_{b a}^{c} T_{c}^{\nu} .
\end{aligned}
$$

Since

$$
\begin{aligned}
D_{b} L_{a}^{\nu} & =D_{b}\left[D_{a} V^{\nu}-V^{\rho} \nabla_{\rho} T_{a}^{\nu}\right] \\
& =D_{b} D_{a} V^{\nu}-\left(D_{b} V^{\rho}\right) \nabla_{\rho} T_{a}^{\nu}-V^{\omega} D_{b} \nabla_{\omega} T_{a}^{\nu},
\end{aligned}
$$

the equation $(5,9)$ reduces to

$$
\Delta K_{b a}^{\prime}=D_{b} L_{a}^{\prime}+\left(\nabla_{\mu} T_{a}^{\nu}\right)\left(\nabla_{b} V^{\mu}-V^{\rho} \nabla_{\rho} T_{b}^{\mu}\right)-Q_{b a}^{c} T_{c}^{\prime}
$$

which proves $(5,7) \mathrm{a}$ and $(5,8)$. The equation $(5,7) \mathrm{b}$ follows from $(5,7)$ a and

$$
\begin{aligned}
\left(T_{b}^{\mu} \nabla_{\mu} V^{\omega} \nabla_{\omega}-V^{\omega} \nabla_{\omega} T_{b}^{\mu} \nabla_{\mu}\right) T_{a}^{\nu} & =\left[L_{b}^{\mu} \nabla_{\mu}+V^{\omega} T_{b}^{\mu}\left(\nabla_{\mu} \nabla_{\omega}-\nabla_{\omega} \nabla_{\mu}\right)\right] T_{a}^{\nu} \\
& =L_{b}^{\mu} \nabla_{\mu} T_{a}^{\nu}-V^{\omega} T_{b}^{\mu} T_{a}^{\lambda} R_{\mu \omega \lambda}^{\nu}
\end{aligned}
$$


6. The field $K_{a_{r} \ldots a_{1}}^{\nu}$. The last theorem may easily be generalized: Theorem (6,1). Put

$$
\begin{aligned}
L_{a_{r} \cdots a_{1}}^{\nu} \equiv & D_{a_{r}} L_{a_{r-1} \cdots a_{1}}^{\prime}+L_{a_{r}}^{\mu} \nabla_{\mu} K_{a_{r-1} \cdots a_{1}}^{\prime} \\
& -\sum_{1}^{r-1} Q_{a_{s} a_{r}}^{c} K_{a_{r-1}}^{\prime} \cdots a_{s+1} c a_{s-1} \cdots a_{1} .
\end{aligned}
$$

Then

$$
\begin{aligned}
L_{a_{r} \cdots a_{1}}^{\prime} \equiv & D_{a_{r}} L_{a_{r-1} \cdots a_{1}}^{\prime}+\left(T_{a_{r}}^{\mu} \nabla_{\mu} V^{\omega} \nabla_{\omega}-V^{\omega} \nabla_{\omega} T_{a_{r}}^{\mu} \nabla_{\mu}\right) K_{a_{r-1} \cdots a_{1}}^{\nu} \\
& -V^{\omega} T_{a_{r}}^{\mu} R_{\omega \mu \lambda}^{\prime} K_{a_{r-1}}^{\lambda} \cdots a_{1}-\sum_{1}^{r-1} Q_{a_{s} a_{r}}^{c} K_{a_{r-1}}^{\nu} \cdots a_{s+1} c_{a_{s-1}} \cdots a_{1}
\end{aligned}
$$

and

$$
\Delta K_{a_{r} \cdots a_{1}}^{\prime}=L_{a_{r} \cdots a_{1}}^{\nu} \quad(r=2,3, \cdots) .
$$

Proof. (The theorem has already been proved for $r=2$.) Let us assume that we have proved it for some $r \geqq 2$. Then we have from $(6,2)$

$$
\begin{aligned}
{ }^{*} K_{a_{r} \cdots a_{1}}^{\nu} & =K_{a_{r} \cdots a_{1}}^{\prime \nu}+\epsilon L_{a_{r} \cdots a_{1}}^{\nu}+\cdots \\
& =K_{a_{r} \cdots a_{1}}^{\nu}+\epsilon\left[L_{a_{r} \cdots a_{1}}^{\nu}+V^{\omega} \partial_{\omega} K_{a_{r} \cdots a_{1}}^{\nu}\right]+\cdots
\end{aligned}
$$

On the other hand

$$
\begin{aligned}
{ }^{*} D_{a_{r+1}}{ }^{*} K_{a_{r} \cdots a_{1}}^{\prime} \doteq \partial_{a_{r+1}}{ }^{*} K_{a_{r} \cdots a_{1}}^{\prime} & +\epsilon\left[V^{\omega}\left(\partial_{\omega} \Gamma_{\lambda \mu}^{\nu}\right) T_{a_{r+1}}^{\mu} K_{a_{r} \cdots a_{1}}^{\lambda}\right. \\
& \left.-\sum_{1}^{r} \cdot W_{a_{s} a_{r+1}}^{\mathrm{c}} K_{a_{r} \cdots a_{s+1} c a_{s-1} \cdots a_{1}}^{\prime}\right]+\cdots
\end{aligned}
$$

and consequently by virtue of $(6,3)$

${ }^{*} K_{a_{r+1} \cdots a_{1}}^{\prime} \doteq \partial_{a_{r+1}} K_{a_{r} \cdots a_{1}}^{\nu}+\epsilon\left\{\partial_{a_{r+1}} L_{a_{r} \cdots a_{1}}^{\nu}\right.$

$$
\begin{aligned}
& +\left(\partial_{a_{r+1}} V^{\omega}\right) \partial_{\omega} K_{a_{r} \cdots a_{1}}^{\nu}+V^{\omega} \partial_{a_{r+1}} \partial_{\omega} K_{a_{r} \cdots a_{1}}^{\nu} \\
& +V^{\omega}\left(\partial_{\omega} \Gamma_{\lambda_{\mu}}^{\nu}\right) T_{a_{r+1}}^{\mu} K_{a_{r}}^{\lambda} \cdots a_{1} \\
& \left.-\sum_{1}^{r} W_{a_{s} a_{r+1}}^{0} K_{a_{r} \cdots a_{s+1} a_{s-1} \cdots a_{1}}^{\prime}\right\}+\cdots
\end{aligned}
$$




$$
\begin{aligned}
\doteq D_{a_{r+1}} K_{a_{r} \cdots a_{1}}^{\prime} & +\epsilon\left\{D_{a_{r+1}} L_{a_{r} \cdots a_{1}}^{\prime}\right. \\
& +\left(\nabla_{a_{r+1}} V^{\rho}\right) \nabla_{\rho} K_{a_{r} \cdots a_{1}}^{\prime} \\
& -\sum_{1}^{r} W_{a_{s} a_{r+1}}^{c} K_{a_{r} \cdots a_{s+1} c a_{s-1} \cdots a_{1}}^{\prime} \\
& \left.+V^{\omega}\left[\left(\partial_{\omega} \Gamma_{\lambda \mu}^{\prime}\right) T_{a_{r+1}}^{\mu} K_{a_{r} \cdots a_{1}}^{\lambda}+\partial_{a_{r+1} \partial_{\omega}} K_{a_{r} \cdots a_{1}}^{\prime}\right]\right\} \\
& +\cdots
\end{aligned}
$$

The tensor $K_{a_{r+1} \ldots a_{1}}^{\nu}$ satisfies the following relation

$(6,5)$

$$
\begin{aligned}
K_{a_{r}+\cdots a}^{\prime \prime}= & K_{a_{r+1} \cdots a_{1}}^{\prime \prime}+\epsilon V^{\omega} \partial_{\omega} K_{a_{r+1} \cdots a_{1}}^{\prime}+\cdots \\
= & K_{a_{r+1} \cdots a_{1}}^{\prime}+\epsilon V^{\omega} \partial_{\omega} D_{a_{r+1}} K_{a_{r} \cdots a_{1}}^{\prime}+\cdots \\
\doteq & K_{a_{r+1} \cdots a_{1}}^{\prime}+\epsilon V^{\omega}\left[\partial_{\omega} \partial_{a_{r+1}} K_{a_{r} \cdots a_{1}}^{\prime} \cdots\right. \\
& +\left(\partial_{\omega} \Gamma_{\lambda_{\mu}}^{\prime}\right) T_{a_{r+1}}^{\mu} K_{a_{r} \cdots a_{1}}^{\lambda} \\
& \left.-\sum_{1}^{r}\left(\partial_{\omega} \Gamma_{a_{s} a_{r+1}}^{e}\right) K_{a_{r} \cdots a_{s+1} \epsilon a_{s-1} \cdots a_{1}}^{\prime}\right]+\cdots
\end{aligned}
$$

Subtracting $(6,5)$ from $(6,4)$ we have

$$
\begin{aligned}
& { }^{*} K_{a_{r+1}}^{\prime} \cdots a_{1}-K_{a_{r+1} \cdots a_{1}}^{\prime \prime} \doteq \epsilon\left[D_{a_{r+1}} L_{a_{r} \cdots a_{1}}^{\prime}\right.
\end{aligned}
$$

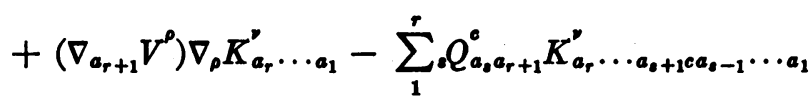

$$
\begin{aligned}
& \left.+V^{\omega}\left(\partial_{a_{r+1}} \partial_{\omega}-\partial_{\omega} \partial_{a_{r+1}}\right) K_{a_{r}}^{\nu} \ldots a_{1}\right]+\cdots \cdot
\end{aligned}
$$

Since

$$
\begin{aligned}
\left(\partial_{a_{r+1}} \partial_{\omega}-\partial_{\omega} \partial_{a_{r+1}}\right) K_{a_{r}}^{\nu} & \cdots a_{1} \\
& =\left[T_{a_{r+1}}^{\mu} \partial_{\mu} \partial_{\omega}-\left(\partial_{\omega} T_{a_{r+1}}^{\mu}\right) \partial_{\mu}-T_{a_{r+1}}^{\mu} \partial_{\omega} \partial_{\mu}\right] K_{a_{r} \cdots a_{1}}^{\nu} \\
& \doteq-\left(\nabla_{\omega} T_{a_{r+1}}^{\mu}\right) \nabla_{\mu} K_{a_{r} \cdots a_{1}}^{\prime},
\end{aligned}
$$

the equation $(6,6)$ reduces to 


$$
\begin{aligned}
& { }^{*} K_{a_{r+1} \cdots a_{1}}^{\prime} \quad K_{a_{r+1} \cdots a_{1}}^{\prime \prime} \doteq \epsilon\left[D_{a_{r+1}} L_{a_{r} \cdots a}^{\prime}\right. \\
& \left.+L_{a_{r+1}}^{\rho} \nabla_{\rho} K_{a_{r} \cdots a_{1}}^{\prime}-\sum_{1}^{r} Q_{a_{s} a_{r+1}}^{c} K_{a_{r} \cdots a_{t+1} c a_{s-1} \cdots a_{1}}^{\prime}\right]+\cdots
\end{aligned}
$$

In this tensor equation we may obviously suppress the dot in $\doteq$ and consequently if we put

$$
\begin{aligned}
L_{a_{r+1}}^{\prime} \cdots a_{1} & D_{a_{r+1}} L_{a_{r} \cdots a_{1}}^{\prime}+L_{a_{r+1}}^{\rho} \nabla_{p} K_{a_{r} \cdots a_{1}}^{\prime} \\
& -\sum_{1}^{\gamma} Q_{a_{s} a_{r+1}}^{c} K_{a_{r} \cdots a_{r+1} c a_{s-1} \cdots a_{1}}^{\prime},
\end{aligned}
$$

we have from $(6,7)$

$$
\Delta K_{a_{r+1} \cdots a_{r}}^{\prime}=L_{a_{r+1} \cdots a_{1}}^{\prime} .
$$

Hence if $(6,2)$ holds for some $r$ then it holds also for $r+1$. But $(6,2)$ holds for $r=2$ and consequently it holds for $r=2,3, \ldots$. The remaining part of the theorem (equation $(6,1) \mathrm{b}$ ) may be proved by means of an equation similar to $(5,10)$

\section{The differential equation $L_{a}^{\nu}=0$.}

THEOREM $(7,1)$. The system of differential equations

$$
L_{a}^{\nu}=0 \text { or } \nabla_{a} V^{\prime}=V^{\lambda} \nabla_{\lambda} T_{a}^{\nu}
$$

is completely integrable and its general solution involves $n$ arbitrary constants.

Proof. We have first

$$
\begin{aligned}
\nabla_{[b} \nabla_{a]} V^{\prime} & =\nabla_{[b} T_{a]}^{\mu} \nabla_{\mu} V^{\prime}=\left(\nabla_{[b} T_{a]}^{\mu}\right) \nabla_{\mu} V^{\nu}+T_{[b}^{\omega} T_{a]}^{\mu} \nabla_{\omega} \nabla_{\mu} V^{\prime} \\
& =-\frac{1}{2} R_{\omega \mu \lambda}^{\nu} V^{\lambda} T_{b a}^{\omega \mu}
\end{aligned}
$$

and by virtue of $(7,1)$

$$
\begin{aligned}
\nabla_{[b} V^{\lambda} \nabla_{|\lambda|} T_{a]}^{\nu} & =\left(\nabla_{[b} V^{\lambda}\right) \nabla_{|\lambda|} T_{a]}^{\prime}+V^{\mu} T_{[b}^{\omega} \nabla_{\mid \omega} \nabla_{\mu \mid} T_{a]}^{\nu} \\
& =V^{\omega}\left[\left(\nabla_{\omega} T_{[b}^{\mu}\right)\left(\nabla_{|\mu|} T_{a]}^{\nu}\right)+T_{[b}^{\mu} \nabla_{\mid \mu} \nabla_{\mid \omega} T_{a]}^{\nu}\right] .
\end{aligned}
$$

On the other hand

$$
0=V^{\omega} \nabla_{\omega} T_{[b}^{\mu} \nabla_{|\mu|} T_{a]}^{\nu}=V^{\omega}\left(\nabla_{\omega} T_{[b}^{\mu}\right) \nabla_{|\mu|} T_{a]}^{\prime}+V^{\omega} T_{[b}^{\mu} \nabla_{\omega \mid} \nabla_{\mu \mid} T_{a]}^{\nu}
$$


and consequently $(7,3)$ reduces to

$$
\nabla_{[b} V^{\lambda} \nabla_{[\lambda \mid} T_{a]}^{\nu}=V^{\omega} T_{[b}^{\mu}\left(\nabla_{\mid \mu} \nabla_{\omega \mid}-\nabla_{\mid \omega} \nabla_{\mu \mid}\right) T_{a]}^{\nu}=V^{\omega} T_{[b a]}^{\mu \lambda} R_{\omega \mu \lambda}^{\nu} .
$$

The integrability conditions of $(7,1)$ are obtained by substituting in

$$
\nabla_{[b} \nabla_{a]} V^{\nu}=\nabla_{[b} V^{\lambda} \nabla_{|\lambda|} T_{a]}^{\nu}
$$

from $(7,2)$ and $(7,4)$. In doing so we obtain

$$
\begin{aligned}
\frac{1}{2} V^{\lambda} T_{b a}^{\omega \mu}\left(-R_{\omega \mu \lambda}^{\nu}-R_{\lambda \omega \mu}^{\nu}+\right. & \left.R_{\lambda \mu \omega}^{\nu}\right) \\
& =-\frac{1}{2} V^{\lambda} T_{b a}^{\omega \mu}\left(R_{\omega \mu \lambda}^{\nu}+R_{\lambda \omega \mu}^{\nu}+R_{\mu \lambda \omega}^{\nu}\right) \equiv 0
\end{aligned}
$$

since

$$
R_{\omega \mu \lambda}^{\prime}+R_{\mu \lambda \omega}^{\prime \prime}+R_{\lambda \omega \mu}^{\prime \prime} \equiv 0
$$

Hence the integrability conditions of $(7,1)$ are identically satisfied and consequently $(7,1)$ is completely integrable and its general solution involves $n$ arbitrary constants.

Theorem $(7,2)$. Let $V_{1}^{\nu}, \cdots, V_{n}^{\nu}$ be $n$ linear independent solutions of $(7,1)$. Then ${ }^{10}$

$$
W^{\prime}=V_{\left[\mathfrak{a} \partial_{\mu}\right.}^{\mu} V_{\mathfrak{e}]}^{\prime} \quad(\mathfrak{a}, \mathfrak{e}=1, \cdots, n ; \mathfrak{a} \neq \mathfrak{e})
$$

is a vector solution of $(7,1)$.

Proof. $W^{\nu}$ is obviously a vector. Hence to prove our theorem we have to show that it satisfies the equation

$$
\partial_{a} W^{\nu}=W^{\lambda} \partial_{\lambda} T_{a}^{\nu}
$$

From $(7,1)$ we have for

$$
\partial_{a} W^{\nu}=\partial_{a} V_{[a}^{\mu} \partial_{\mu} V_{\mathrm{e}]}^{\nu}=V_{[a}^{\lambda}\left(\partial_{\lambda} T_{a}^{\mu}\right) \partial_{\mu} V_{\mathrm{e}]}^{\nu}+V_{[a}^{\mu} \partial_{a} \partial_{\mu} V_{\mathrm{e}]}^{\nu} \text {. }
$$

On the other hand $\left(\partial_{a} \partial_{\mu}-\partial_{\mu} \partial_{a}\right) V_{\mathrm{e}}^{\nu}=-\left(\partial_{\mu} T_{a}^{\lambda}\right) \partial_{\lambda} V_{\mathrm{e}}^{\nu}$ and consequently $(7,7)$ reduces to

$$
\begin{aligned}
\partial_{a} W^{\nu} & =\left(\partial_{\lambda} T_{a}^{\mu}\right)\left(V_{[a}^{\lambda} \partial_{\mu} V_{\mathrm{e}]}^{\nu}-V_{[a}^{\lambda} \partial_{\mu} V_{\mathrm{e}]}^{\nu}\right)+V_{[a}^{\mu} \partial_{\mu} \partial_{a} V_{\mathrm{e}]}^{\nu} \\
& =V_{[a}^{\mu} \partial_{\mu} V_{\mathrm{e}]}^{\lambda} \partial_{\lambda} T_{a}^{\nu}=V_{[a}^{\mu}\left(\partial_{\mu} V_{\mathrm{e}]}^{\lambda}\right) \partial_{\lambda} T_{a}^{\nu}=W^{\lambda} \partial_{\lambda} T_{a}^{\nu} .
\end{aligned}
$$

${ }^{10} \mathrm{It}$ is assumed that in the equations $(7,5)-(7,8)$ the brackets [ ] do not affect the Greek and Latin indices. 
REMARK. The theorem $(7,2)$ shows that the set of all generators $V^{\lambda} \partial_{\lambda}$ of infinitesimal transformations $(2,1)$ is closed under the operations of taking linear combination and forming commutators. Hence these generators form a Lie algebra.

8. Special cases.

DefinItION $(8,1)$. We say that a transformation $(2,1)$ reproduces the family $\left(V_{m}\right)$ if

$$
\Delta K_{a_{r} \cdots a_{1}}^{\nu}=0 \quad\left(r=1,2, \cdots ; K_{a}^{\nu} \equiv T_{a}^{\nu}\right) .
$$

Theorem $(8,1)$. A necessary and sufficient condition that $\left(V_{m}\right)$ be reproduced under $(2,1)$ is: $V^{v}$ is a solution of

$$
L_{a}^{*}=0 .
$$

PRoof. Let $\left(V_{m}\right)$ be reproduced by $(2,1)$. Then from the first of $(8,1)$ we have $(8,2)$ by virtue of $(3,1)$ c. Conversely, if $(8,2)$ is satisfied, the following equations hold (cf. $(3,4) \mathrm{c}$ and $(4,8)$ )

$$
\begin{aligned}
& \text { (a) }{ }^{*} g_{a b}=g_{a b}^{\prime}+\cdots, \\
& \text { (b) } \Gamma_{a b}^{c}=\Gamma_{a b}^{c \prime}+\cdots .
\end{aligned}
$$

Consequently we have from $(4,1) \mathrm{c}$ and $(8,3) \mathrm{b}$

(c) $* \Gamma_{a b}^{c}={ }^{\prime} \Gamma_{a b}^{c}+\epsilon Q_{a b}^{c}+\cdots$.

On the other hand we get by virtue of $(8,3) \mathrm{a}$ and $(8,3) \mathrm{b}$

$$
\text { (d) } \quad * \Gamma_{a b}^{c}={ }^{\prime} \Gamma_{a b}^{c}+\cdots \text {. }
$$

Comparing $(8,3)(c)$ and $(d)$ we have

$$
Q_{a b}^{c}=0 .
$$

The equations $(8,2),(8,4)$ together with $(6,2)$ and $(6,1)$ a lead to $(8,1)$ for $r=2,3, \cdots$.

In the next theorem we shall consider a family $\left(V_{m}\right)$ of totally geodesic subspaces, that is, a family for which

$$
K_{a b}^{\prime}=0
$$

and we prove first the following lemma.

Lemma $(8,1)$. If $(8,5)$ holds, then $W_{a b d}(c f$. the equation $(4,2))$ satisfies

1 This equation imposes some conditions on the structure of the large space $V_{n}$ (cf. the equation $(8,12)$ ). 
the following equation

$$
W_{b a d} \equiv W_{a b d}=P_{b d a}^{c} W_{c}+D_{a} D_{b} W_{d}
$$

where $P_{b d a}^{c}$ is the curvature tensor of $V_{m}$ and $W_{c} \equiv T_{c}^{\lambda} V_{\lambda}$.

Proof. Let $W^{v}\left(N^{\nu}\right)$ be the tangential (normal) component of $V^{v}$

$$
V^{\prime}=W^{\prime}+N^{\prime}, \quad W^{\prime}=V^{\lambda} T_{\lambda}^{c} T_{c}^{\prime}, \quad N_{\nu} T_{c}^{\prime}=0 .
$$

From $(3,5)$ we have

$$
\begin{aligned}
W_{a b}=T_{(a}^{\lambda} T_{b)}^{\mu} \nabla_{\lambda} V_{\mu} & =T_{(a b)}^{\lambda_{\mu}} \nabla_{\lambda} W_{\mu}+T_{(a b)}^{\lambda_{\mu}} \nabla_{\lambda} N_{\mu} \\
& =D_{(a} W_{b)}-N_{\mu} \nabla_{(a} T_{b)}^{\mu} .
\end{aligned}
$$

If $(8,5)$ holds then

$$
0=N_{\mu} D_{a} T_{b}^{\mu}=N_{\mu}\left(\nabla_{a} T_{b}^{\mu}-\Gamma_{b a}^{c} T_{c}^{\mu}\right)=N_{\mu} \nabla_{a} T_{b}^{\mu}
$$

and $(8,8)$ a reduces to

$$
\left.W_{a b}=D_{(a} W_{b}\right) .
$$

Consequently, we get from $(4,2) a$

$(8,9) \mathrm{a} \quad W_{a b d}=D_{[a} D_{d]} W_{b}+D_{[b} D_{d]} W_{a}+D_{[b} D_{a]} W_{d}+D_{a} D_{b} W_{d}$.

Because for any vector $P_{\mathbf{b}}$ whatsoever

$$
\begin{gathered}
D_{[a} D_{d]} P_{b}+D_{[d} D_{b]} P_{a}+D_{[b} D_{a]} P_{d}=0 \\
D_{[b} D_{d]} P_{a}=1 / 2 P_{b d a}^{c} P_{c}
\end{gathered}
$$

where $P_{d b a}^{c}$ is the curvature tensor of $V_{m},(8,9)$ a reduces to $(8,6)$.

Definition $(8,2)$. If $A=0$ is an analytic expression for a property of $\left(V_{m}\right)$, then we say that $(2,1)$ preserves this property if $\Delta A=0$.

Theorem $(8,2)$. Let $\left(V_{m}\right)$ be a family of totally geodesic subspaces. $A$ necessary and sufficient condition that this property be preserved by $(2,1)$ is: The vector $V^{v}$ is a solution of any one of the following equations

$$
\begin{aligned}
& \text { (a) } L_{a b}^{\prime}=0, \\
& \text { (b) } D_{b} D_{a} V^{\prime}-V^{\omega} T_{b a}^{\mu \lambda} R_{\omega \mu \lambda}^{\prime}-W_{b a}^{c} T_{c}^{\prime}=0, \\
& \text { (c) } D_{b} D_{a} N^{\prime}-N^{c} T_{b a}^{\mu \lambda} R_{\omega \mu \lambda}^{\prime}=0,
\end{aligned}
$$

where in the last equation

$$
N^{\prime}=V^{\lambda}\left(\delta_{\lambda}^{\prime}-T_{c}^{\prime} T_{\lambda}^{c}\right)
$$




\section{A sufficient but not necessary condition is:}

The vector $V^{v}$ is a solution of $(8,2)$. If $(8,10)$ but not $(8,2)$ is satisfied by $V^{p}$, then $\left(V_{m}\right)$ is not reproduced. If $(8,2)$ holds, then $(8,10)$ is satisfied and $\left(V_{m}\right)$ is reproduced.

Proof. According to our assumption the transformation $(2,1)$ carries a family $\left(V_{m}\right)$ in a family $\left(V_{m}^{*}\right)$. Hence in order to prove our theorem we have only to look for conditions that the equation $K_{a b}^{\nu}=0$ be satisfied. The necessity and sufficiency of $(8,10)$ a follows at once from $(5,8)$. If $(8,5)$ is satisfied, then we have

$$
K_{a b}^{\prime \prime}=K_{a b}^{\prime}+\epsilon V^{\lambda} \partial_{\lambda} K_{a b}^{\prime}+\cdots=0+\cdots
$$

and consequently

$$
\Delta K_{a b}^{\prime}=(\tau-\omega) K_{a b}^{\prime}=\tau K_{a b}^{\prime} .
$$

Hence, we have from $(8,11) b,(5,8)$ and $(5,2)$ that in our case a necessary and sufficient condition for $\Delta K_{a b}^{p}=0$ is that $V^{v}$ be a solution of $(8,10) \mathrm{b}$ and this equation is (in our case) equivalent to $(8,10) \mathrm{a}$. On the other hand, the integrability conditions of $(8,5)$ are

$$
P_{c b a}^{d} T_{d}^{\prime}=T_{c b a}^{\omega \mu \lambda} R_{c \omega \lambda \lambda}^{\prime}
$$

and consequently $T_{c b a}^{\omega \mu \lambda} R_{\omega \mu \lambda}^{\nu}$ is in $\left(V_{m}\right)$. If we decompose $V^{\nu}$ according to $(8,7)$, then we have from $(8,12)$

$$
\begin{aligned}
-V^{\omega} T_{b a}^{\mu \lambda} R_{\omega \mu \lambda}^{\nu} & =-W^{c} T_{c b a}^{\omega \mu \lambda} R_{\omega \mu \lambda}^{\nu}-N^{\omega} T_{b a}^{\mu \lambda} R_{\omega \mu \lambda}^{\prime} \\
& =-W^{c} P_{c b a}^{d} T_{d}^{\nu}-N^{\omega} T_{b a}^{\mu \lambda} R_{\omega \mu \lambda \lambda}^{\nu} .
\end{aligned}
$$

On the other hand, if $(8,5)$ holds then

$$
D_{b} D_{a} V^{\prime}=D_{b} D_{a} W^{c} T_{c}^{\prime}+D_{b} D_{a} N^{\prime}=\left(D_{b} D_{a} W^{c}\right) T_{c}^{\prime}+D_{b} D_{a} N^{\prime}
$$

Furthermore, if $(8,5)$ holds we have according to $(8,6)$

$$
\begin{aligned}
-W_{b a}^{c} T_{c}^{\prime} & =-P_{b d a}^{c} W_{e g}^{d c} T_{c}^{\prime}-\left(D_{a} D_{b} W^{c}\right) T_{c}^{\nu} \\
& =\left(P_{c a b}^{c} W^{c}-D_{a} D_{b} W^{c}\right) T_{c .}^{\prime} 12
\end{aligned}
$$

Comparing $(8,10) \mathrm{b}$ with $(8,13)$ we have

$$
\begin{aligned}
& D_{b} D_{a} V^{\prime}-V^{\omega} T_{b a}^{\mu \lambda} R_{\omega \mu \lambda}^{\prime}-W_{b a}^{c} T_{c}^{\prime} \\
&= T_{d}^{\prime}\left[D_{b} D_{a} W^{d}-W^{c} P_{c b a}^{d}+P_{c a b}^{d} W^{c}-D_{a} D_{b} W^{d}\right] \\
&+D_{b} D_{a} N^{\nu}-N^{\omega} T_{b a}^{\mu \lambda} R_{\omega \mu \lambda}^{\prime}=0 .
\end{aligned}
$$

12 We use here the well known identities $P_{\text {bace }}=P_{a c b c}=-P_{\text {aabce. }}$. 
Since

$$
\begin{aligned}
\left(D_{b} D_{a}-D_{a} D_{b}\right) W^{d} & =-P_{b a c}^{d} W^{c} \\
& =P_{a b c}^{d} W^{c}=-\left(P_{b c a}^{d}+P_{c a b}^{d}\right) W^{c}
\end{aligned}
$$

we obtain from $(8,14) a$

$(8,14) \mathrm{b} \quad D_{b} D_{a} V^{\nu}-V^{\omega} T_{b a}^{\mu \lambda} R_{\omega \mu \lambda}^{\nu}-W_{b a}^{c} T_{c}^{\nu}=D_{b} D_{a} N^{\nu}-N^{\omega} T_{b a}^{\mu \lambda} R_{\omega \mu \lambda}^{\nu}$

and this equation proves the statement about $(8,10) c$. The last part of the theorem follows easily from Theorem $(8,1)$.

REMARK I. The statement about the equation $(8,10) \mathrm{b}$ is a generalization of Levi-Civita's result for $m=1 .{ }^{13}$

REMARK II. Let $m=1, n=2$. Then

$$
\begin{gathered}
R_{\omega \mu \lambda}^{\prime}=2 K g_{\lambda[\omega} \delta_{\mu]}^{\nu}, \\
N^{\omega} T_{1}^{\mu} R_{1}^{\lambda} R_{\omega \mu \lambda}^{\prime}=-K N^{\nu} T \quad\left(T=g_{\lambda \mu} T_{1}^{\lambda} T_{1}^{\mu}\right) .
\end{gathered}
$$

If $\eta^{1} \equiv s, s$ being the arc of $V_{1}$, then $D_{1}=T_{1}^{\lambda} \nabla_{\lambda}$ and $T=1$. Moreover, if $j^{v}$ is the unit vector in the direction of $D_{1} T_{1}^{\nu}$, then

$$
D_{1} T_{1}^{\prime}=j^{\prime \prime} k, \quad D_{1} j^{\prime}=-k T_{1}^{\prime},
$$

where $k$ is the curvature of $V_{1}$. Because we may put $N^{v}=y j^{\nu}, y$ being a factor of proportionality, we have on account of $(8,16)$

$$
D_{1} N^{\prime}=y^{\prime} j^{\prime}-y k T_{1}^{\prime} \text {. }
$$

Consequently

$$
D_{1}^{2} N^{\prime}=y^{\prime \prime} j-2 y^{\prime} k T_{1}^{\prime}-y k^{\prime} T_{1}^{\prime}-y k^{2} j^{\prime \prime} .
$$

If $\left(V_{1}\right)$ consists of geodesic lines, we have $k=0$ and

$$
j_{n} D_{1}^{2} N^{\prime \prime}=y^{\prime \prime} \text {. }
$$

On the other hand, we obtain from $(8,15)$ for $\eta^{1}=s$

$$
j_{v} N^{\omega} T_{1}^{\mu} T_{1}^{\lambda} R_{\omega \mu \lambda}^{\nu}=-K y .
$$

Comparing $(8,10) \mathrm{c}$ and $(8,18)$ we have

$$
y^{\prime \prime}+K y=0,
$$

${ }^{13}$ Levi-Civita, T., Sullo scostamento geodetico (Bollettino della Unione Matematica Italiana (1926) pp. 1-4) and Sur l'écart géodésique (Math. Ann. vol. 97 (1926) pp. 292-320). For more general metric spaces cf. also Hlavaty, V., Les courbes de la varieté generale d $n$ dimensions (Mémorial des Sciences Mathématiques, no. 42). 
which is the well known equation found by Jacobi.

\section{BIBLIOGRAPHY}

1. J. A. Schouten, On infinitesimal deformations of $V_{m}$ in $V_{n}$, Proc. K. Akademie van Wetenschappen vol. 31 (1928) pp. 208-218.

2. E. Bortolotti, Scostamento geodetico e sue generalizzazioni, Giornale di matematiche vol. 66 (1928) pp. 153-191.

3. A. G. Walker, On small deformation of sub-spaces of a flat space, Proceedings of the Edinburgh Mathematical Society vol. 3 (1932) pp. 77-86.

4. J. A. Schouten and E. R. van Kampen, Beitrdge zur theorie der deformation, Prace Matematyczno-Fizyczne Warszawa vol. 41 (1933) pp. 1-19.

5. H. A. Hayden, Infinitesimal deformations of sub-spaces in a general metrical space, Proc. London Math. Soc. vol. 37 (1934) pp. 416-440.

6. - Infinitesimal deformation of an $L_{m}$ in an $L_{n}$, Proc. London Math. Soc. vol. 41 (1936) pp. 332-336.

7. J. A. Schouten and D. J. Struik, Einfilhrung in die neueren Methoden der Differentialgeometrie, vol. 1, Groningen, 1935, p. 151.

8. E. T. Davies, On the deformation of a subspace, J. London Math. Soc. vol. 11 (1936) pp. 295-301.

9. - On the second and third fundamental forms of a subspace, J. London Math. Soc. vol. 12 (1937) pp. 290-295.

10. P. Dienes and E. T. Davies, On the infinitesimal deformations of tensor submanifolds, Journal de Mathématiques vol. 16 (1937) pp. 111-150.

11. K. Yano, Sur les déformations infinitésimales des sous-espaces dans un espace affine, Proc. Imp. Acad. Tokyo vol. 21 (1945) pp. 248-260.

12. - Sur la déformation infinitésimale tangentielle d'un sous-espace, Proc. Imp. Acad. Tokyo vol. 21 (1945) pp. 261-268.

INDIANA UNIVERSITY 